\title{
Gluon propagator and confinement scenario in Coulomb gauge
}

\author{
Attilio Cucchieri ${ }^{\mathrm{a} *}$ and Daniel Zwanziger ${ }^{\mathrm{b} \dagger}$ \\ aIFSC São Paulo University, C.P. 369 CEP 13560-970, São Carlos (SP), Brazil \\ bPhysics Department, New York University, New York, NY 10003, USA
}

We present numerical results in $S U(2)$ lattice gauge theory for the instantaneous part of the gluon propagator in Coulomb gauge $D_{44 \text {,inst }}=V_{\text {coul }}(R) \delta(t)$. Data are taken on lattice volumes $24^{4}$ and $28^{4}$ for 7 values of $\beta$ in the interval $2.2 \leq \beta \leq 2.8$. The data are confronted with the confinement scenario in Coulomb gauge. They are consistent with a linearly rising color-Coulomb potential $V_{\text {coul }}(R)$.

\section{CONFINEMENT SCENARIO IN COULOMB GAUGE}

A particularly simple confinement scenario [1] is available in the minimal Coulomb gauge. It attributes confinement of color to the long range of the color-Coulomb potential $V_{\text {coul }}(R)$. This quantity is the instantaneous part of the 4-4 component of the gluon propagator, $D_{\mu \nu}(x) \equiv\left\langle g A_{\mu}(x) g A_{\nu}(0)\right\rangle$, namely $D_{44}(\mathbf{x}, t)=$ $V_{\text {coul }}(|\mathbf{x}|) \delta(t)+P(\mathbf{x}, t)$. The vacuum polarization term $P(\mathbf{x}, t)$ is less singular than $\delta(t)$ at $t=0$. Since $A_{4}$ couples universally to color charge, the long range of $V_{\text {coul }}(R)$ suffices to confine all color charge. It was conjectured that it is linearly rising at large $R, V_{\text {coul }}(R) \sim-\sigma_{\text {coul }} R$. If an external quark-antiquark pair is present, the physical potential $V_{\mathrm{W}}(R)$ between them may be extracted from a Wilson loop. The color-Coulomb potential contributes the term $-C V_{\text {coul }}(R)$ directly to the Wilson loop, where $C=\left(N^{2}-1\right) /(2 N)$ in $\mathrm{SU}(\mathrm{N})$ gauge theory with external quarks in the fundamental representation. The minus sign occurs because the antiquark has opposite charge to the quark. The vacuum-polarization term is screening, and one expects that $V_{\mathrm{W}}(R)$ is bounded above by this term asymptotically at large $R$, $V_{\mathrm{W}}(R) \leq-C V_{\text {coul }}(R)$. If $V_{\mathrm{W}}(R)$ is also linearly rising, $V_{\mathrm{W}}(R) \sim \sigma R$, where $\sigma$ is the conventional

\footnotetext{
*Research supported by FAPESP, Brazil (Project No. 00/05047-5).e-mail address: attilio@if.sc.usp.br

${ }^{\dagger}$ Presented talk. Research supported in part by National Science Foundation, grant no. PHY-0099393. e-mail address: daniel.zwanziger@nyu.edu
}

string tension, we get $\sigma \leq C \sigma_{\text {coul }}$. If dynamical quarks are present, the string "breaks" at some radius $R_{b}$, and the conventional asymptotic string tension vanishes, $\sigma=0$. String-breaking is easily explained in the Coulomb-gauge confinement scenario if $V_{\text {coul }}(R)$ is linearly rising even in the presence of dynamical quarks, as was also conjectured [1]. For, if so, it is energetically preferable to polarize a pair of sea quarks from the vacuum.

Here we report the confrontation of this scenario with the numerical data for $V_{\text {coul }}(R)$ for the case of pure gluodynamics.

\subsection{Relation of $V_{\text {coul }}$ to $\alpha_{s}$}

We may identify $V_{\text {coul }}(R)$ with the phenomenological potential that is the starting point for QCD bound state calculations [2]. The identification of a phenomenological potential with the instantaneous part of the gluon propagator, a fundamental quantity in the gauge theory, is possible because, remarkably, $V_{\text {coul }}(R)$ is a renormalization-group invariant, and thus scheme-independent, so it is independent of the cut-off $\Lambda$ and of the renormalization mass $\mu$. This follows from the non-renormalization of $g A_{4}$, as expressed by the identity $g_{(0)} A_{4}^{(0)}=$ $g_{(r)} A_{4}^{(r)}$, where 0 and $r$ refer to unrenormalized and renormalized quantities in the Coulomb gauge [1]. This identity has no direct analog in a Lorentz-covariant gauge. Because of the schemeindependence of $V_{\text {coul }}(R)$, its Fourier transform $\widetilde{V}_{\text {coul }}(\mathbf{k})$ provides a scheme-independent definition for the running coupling constant of QCD, 
Table 1

Values of fitting parameters from formula (11).

\begin{tabular}{lccccc}
\hline$\beta$ & $A$ & $B$ & $\Lambda$ & $W^{2}$ & $\chi^{2} /$ dof \\
\hline 2.2 & $7.38 \pm 0.35$ & $8.15 \pm 15.58$ & $8.83 \pm 19.34$ & $9.91 \pm 19.01$ & 0.81 \\
2.3 & $5.81 \pm 0.30$ & $24.57 \pm 83.41$ & $32.57 \pm 52.59$ & $17.26 \pm 27.18$ & 0.72 \\
2.4 & $6.60 \pm 0.64$ & $6.72 \pm 10.15$ & $7.70 \pm 15.90$ & $3.99 \pm 6.03$ & 0.20 \\
2.5 & $7.45 \pm 0.47$ & $6.00 \pm 2.65$ & $6.16 \pm 2.58$ & $3.06 \pm 1.26$ & 0.05 \\
2.6 & $8.23 \pm 1.48$ & $8.61 \pm 1.68$ & $4.53 \pm 1.93$ & $3.84 \pm 0.34$ & 0.07 \\
2.7 & $11.30 \pm 0.81$ & $7.73 \pm 0.47$ & $6.87 \pm 0.67$ & $3.64 \pm 0.15$ & 0.07 \\
2.8 & $8.18 \pm 2.85$ & $10.38 \pm 0.46$ & $0.58 \pm 0.08$ & $0.06 \pm 0.05$ & 0.19 \\
\hline
\end{tabular}

$\mathbf{k}^{2} \widetilde{V}(\mathbf{k})=x_{0} g_{\text {coul }}^{2}(|\mathbf{k}|)$, and of $\alpha_{s} \equiv \frac{g^{2}\left(\mathbf{k} / \Lambda_{\text {coul }}\right)}{4 \pi}$. Here $x_{0}=\frac{12 N}{11 N-2 N_{f}}$, and $\Lambda_{\text {coul }}$ is a finite QCD mass scale [3].

\section{NUMERICAL STUDY OF $V_{\text {coul }}$}

\subsection{Method}

We have previously studied 4 both space- and time-components of the gluon propagator, $D_{i j}^{\operatorname{tr}}(\mathbf{k})$ and $D_{44}(\mathbf{k})=\tilde{V}_{\text {coul }}(\mathbf{k})$ at equal time, at $\beta=2.2$, on various lattice volumes, $14^{4}$ to $30^{4}$, in the minimal Coulomb gauge. This gauge is achieved numerically by (i) maximizing $\sum_{x, i=1}^{3} \operatorname{Tr}^{g} U_{x, i}$ with respect to all local gauge transformations $g(x)$, where the sum is on all horizontal or spatial links, and then (ii) maximizing $\sum_{x} \operatorname{Tr}^{g} U_{x, 4}$ with respect to all $\mathbf{x}$-independent but $x_{4}$-dependent gauge transformations $g\left(x_{4}\right)$ where the sum is on all vertical or time-like links. This makes the 3 -vector potential $A_{i}$, for $i=1,2,3$ transverse, $\partial_{i} A_{i}=0$, so $A_{i}=A_{i}^{\text {tr }}$.

In this gauge, the horizontal link variables $U_{x, i}$, for $i=1,2,3$ are as close to the identity as possible, but the vertical variables $U_{x, 4}$ are much further from the identity. Not surprisingly, we found that, whereas $D_{i j}^{\operatorname{tr}}(\mathbf{k})$ gave values that could be reasonably extrapolated to the continuum, this was not true for $D_{44}(\mathbf{k})$. In order to remedy this, in the present study we extended our investigation of $D_{44}(\mathbf{k})$ to $\beta=2.2,2.3, \ldots, 2.8$, on lattice volumes $24^{4}$ and $28^{4}$. We have also determined $V_{\text {coul }}(R)$ numerically by 2 quite different methods. Method I relies on the standard formula $D_{44}(\mathbf{x}, t)=V_{\text {coul }}(|\mathbf{x}|) \delta(t)+P(\mathbf{x}, t)$. Method II relies on the lattice analog of the continuum formula that is obtained [3] by integrating out $A_{4}$ namely, $V(|\mathbf{x}-\mathbf{y}|)=\left\langle\left.\left(M^{-1}\left(-\nabla^{2}\right) M^{-1}\right)\right|_{\mathbf{x}, \mathbf{y}}\right\rangle$. Here $M(\mathbf{A})=-\nabla \cdot \mathbf{D}(\mathbf{A})$ is the 3 -dimensional Faddeev-Popov operator, and $\mathbf{D}(\mathbf{A})=\nabla+\mathbf{A} \times$ is the gauge-covariant derivative. Method II requires numerically inverting the lattice FaddeevPopov matrix to obtain $M^{-1}(\mathbf{A})_{\mathbf{x}, \mathbf{y}}$, but it has the advantage that $D_{44}(\mathbf{k})$ is expressed entirely in terms of the spatial link variables $U_{x, i}$ for $i=1,2,3$, that are close to the identity. Moreover, method II involves only the horizontal link variables that lie within a single time slice, so it is independent of the gauge fixing (ii) on vertical links. Thus it measures a truly instantaneous quantity. We found that method I did not exhibit scaling in the above range of $\beta$, and we report here only the result of method II.

\subsection{Results}

For each $\beta$ we have 100 configurations on volume $V=24^{4}$ and 50 configurations on volume $V=28^{4}$. Runs have been done on the PC clusters at the Physics Department of New York University and at the IFSC of São Paulo University.

In figure 1 we plotted the results for $\beta=2.2$, 2.5 , and 2.8 respectively. The horizontal axis measures $\mathbf{k}^{2}=4 a^{-2} \sin ^{2}(n \pi / L)$, for $L=24,28$, rescaled to physical units by setting the physical string tension equal to $\sigma=(0.44 \mathrm{GeV})^{2}$ and using Table 3 of $[5]$, so the lattice spacing at $\beta=2.2, \ldots, 2.8$ is $\left(\right.$ in $\left.\mathrm{GeV}^{-1}\right) a=1.066,0.839$, $0.605,0.433,0.309,0.231,0.165$, respectively. The vertical axis measures $|\mathbf{k}|^{4} \tilde{V}_{\text {coul }}(\mathbf{k})$ in phys- 
ical units, so string tension may be read off from the vertical intercept (see below). Finite-volume artifacts are clearly visible at low momentum. To control these, in our fits we have dropped those low-momentum points for which appreciably different values are obtained at volumes $24^{4}$ and $28^{4}$ and, for the fit, we used only data points obtained at $V=28^{4}$.

A simple parametrization of $V_{\text {coul }}(R)$ would be $-V_{\text {coul }}(R)=\sigma_{\text {coul }} R-c / R$, which has the Fourier transform $\tilde{V}_{\text {coul }}(\mathbf{k})=8 \pi \sigma_{\text {coul }} /|\mathbf{k}|^{4}+4 \pi c /|\mathbf{k}|^{2}$. We have used the fitting formulas

$$
\begin{aligned}
& |\mathbf{k}|^{4} \tilde{V}_{\text {coul }}(\mathbf{k})=A+\frac{B \mathbf{k}^{2}}{W^{2}+\ln \left(1+\mathbf{k}^{2} / \Lambda^{2}\right)} \\
& |\mathbf{k}|^{4} \tilde{V}_{\text {coul }}(\mathbf{k})=A+B \mathbf{k}^{2} \\
& |\mathbf{k}|^{4} \tilde{V}_{\text {coul }}(\mathbf{k})=A|\mathbf{k}|^{s}+B \mathbf{k}^{2} .
\end{aligned}
$$

Fit (11) has the asymptotic behavior at large $\mathbf{k}$, consistent with the 1-loop $\beta$-function. We report in Table 1 the values of the parameters from fit (1).

The most striking feature of the data is the finite intercepts $A$. This is consistent with a finite string tension $\sigma_{\text {coul }}=A /(8 \pi)$. It scales rather nicely, varying from $A=7.38 \pm 0.35 \mathrm{GeV}^{2}$ at $\beta=$ 2.2 to $A=8.18 \pm 2.85 \mathrm{GeV}^{2}$ at $\beta=2.8$, but with considerable variation in between. From the lowest and highest values, $A=5.8$ and $A=11.3$, we get respectively $\sigma_{\text {coul }}=(0.48 \mathrm{GeV})^{2}$ and $\sigma_{\text {coul }}=$ $(0.67 \mathrm{GeV})^{2}$. The inequality $\sigma \leq(3 / 4) \sigma_{\text {coul }}$ for $\mathrm{SU}(2)$, with $\sigma=(0.44 \mathrm{GeV})^{2}$, reads for these values: $0.44 \leq 0.42(2)$ and $0.44 \leq 0.58(3)$. We appear to be at or near saturation.

\section{REFERENCES}

1. D. Zwanziger, Nucl. Phys. B518 (1998) 237.

2. A. Szczepaniak et al., Phys. Rev. Lett. 76 (1996) 2011; D. G. Robertson et al., Phys. Rev. D59 (1999) 074019; A. Szczepaniak and E. S. Swanson, hep-ph/0107078.

3. A. Cucchieri and D. Zwanziger, Phys. Rev. D65 (2001) 014002.

4. A. Cucchieri and D. Zwanziger, Phys. Rev. D65 (2001) 014001; Phys. Letts. B524 (2002) 123.

5. J. Fingberg et al., Nucl. Phys. B392 (1993) 493.
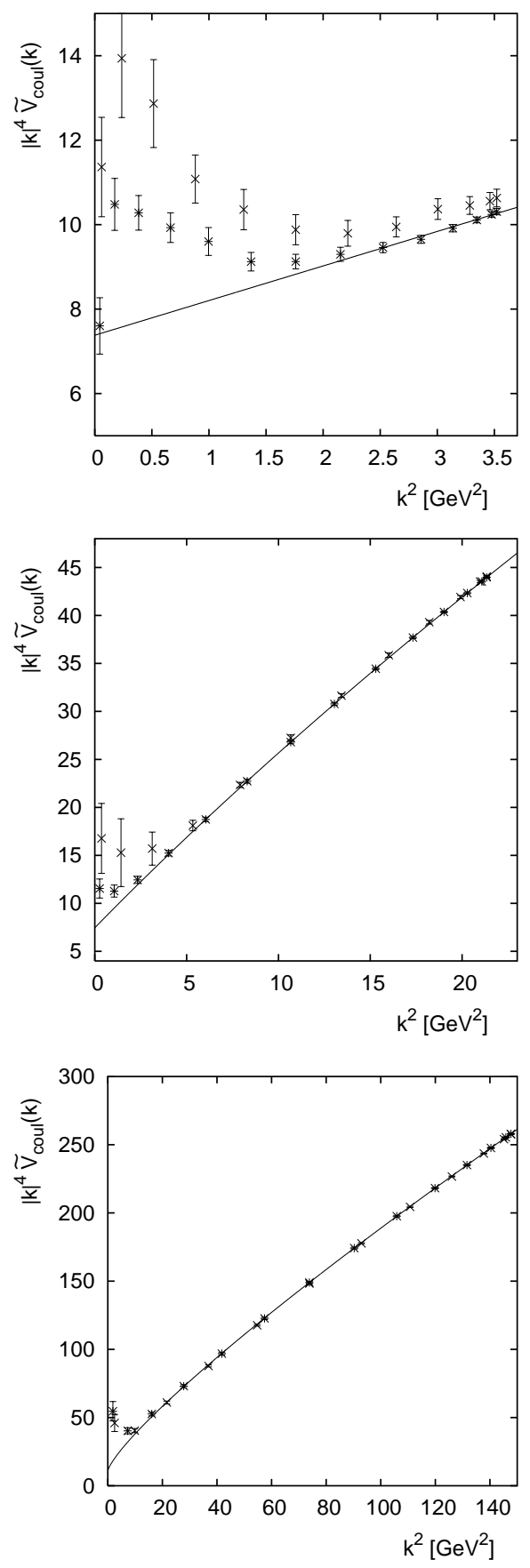

Figure 1. Fit of $|\mathbf{k}|^{4} \tilde{V}_{\text {coul }}(\mathbf{k})$ using eq. (11). Data are for lattice volumes $V=24^{4}(\times)$ and $V=28^{4}$ $(*)$ and $\beta=2.2,2.5$ and 2.8 from top to bottom. 